\title{
A Crystal Plasticity FE Analysis Considering Mechanically Induced Martensitic Phase Transformation
}

\author{
by Seiichiro Tsutsumi**, Riccardo Fincato**, Kazushi Ueda*** and Kenjiro Terada****
}

\begin{abstract}
One of the most challenging topic nowadays for the steel manufacturers is the investigation of the role of the retained austenitic grains inside a martensitic body. The phase transformation of the former, in fact, has been proved to alter the material properties in terms of toughness, ductility, resistance and also it has be indicated as one of the probable causes for the crack initiation. The work presented in this paper aims to study this phenomena by means of a thermodynamically based single crystal constitutive model, coupling the crystallographic slip together with the phase transformation.
\end{abstract}

Key Words: Crystal Plasticity, Martensitic Phase Transformation, Retain Austenite, FE analyses.

\section{Introduction}

Depending on the carbon content of the austenite, the martensite start temperature $\left(\mathrm{M}_{\mathrm{s}}\right)$ and the finish one $\left(\mathrm{M}_{\mathrm{f}}\right)$ can be sensibly altered. In particular, for a high level of carbon, the previous two temperatures are depressed, leading to difficulties in converting all of the austenite to martensite. When this condition is satisfied it is possible to have retained austenite, which may be either extremely non-desirable or desirable depending on the nature of the steel component and on its operative use.

It is also important to know the percentage and the distribution of the retain austenite since the transformation from metastable austenitic phase to martensitic phase has been considered to change the material response ${ }^{1)}$, but more importantly, to decrease the fracture toughness of materials ${ }^{2}$. It has, in fact, been proved that they can have an effect on the local stress and strain distributions through the elongation and volume change during the martensitic phase transformation, accompanied with the lattice-invariant shear.

In this study, local stress distribution at a crystal level has been analyzed by means of FE numerical simulations adopting the crystal plasticity theory and the mechanically induced martensitic transformation (MIMT) under a thermodynamically based single crystal constitutive model. The kinetics of the transformation, in terms of the definition of the orientation of the relationship between the parent austenitic and the martensitic variants, is modeled by taking into consideration the well-known

${ }^{*}$ Received: 2014.11.28

** Member, Joining and Welding Research Institute, Osaka University.

${ }^{* * *}$ Student Member, Joining and Welding Research Institute, Osaka University.

**** International Research Institute of Disaster Science (IRIDeS), Tohoku University.
Kurdjumov-Sachs (KS) relationship. The effect of mechanically induced martensitic transformation on the local stress distribution is discussed under uniaxial extension condition.

\section{Constitutive model}

One of the principal variables to consider when dealing with the finite strain theory is the deformation gradient, which creates a map (see Fig. 1) between the points coordinates of the deformed (current) configuration and the ones of the undeformed (reference).

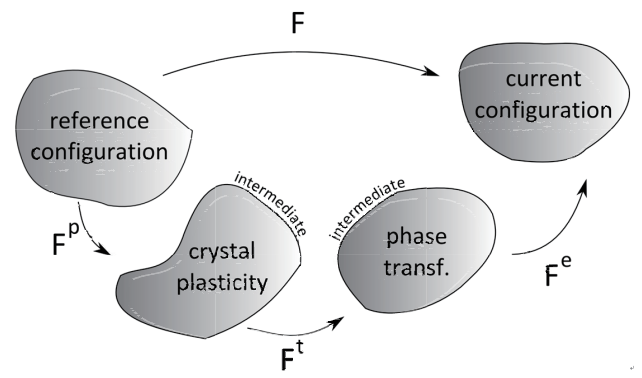

Fig. 1 Multiplicative decomposition of the deformation gradient.

The constitutive model adopted in the present study consider a multiplicative decomposition of the total deformation gradient into three parts: an elastic one $\mathbf{F}^{e}$ (which describes the distortion of the crystal lattice), the deformation caused by the austenite-martensitic transformation process $\mathbf{F}^{t}$ and finally the irreversible plastic slip on the crystallographic planes $\mathbf{F}^{p}$ :

$$
\mathbf{F}=\mathbf{F}^{e} \mathbf{F}^{t} \mathbf{F}^{p}
$$

The position of the transformation term can vary depending on the different authors' approach, in this paper we adopted the one proposed by Idesman et al. ${ }^{3)}$ and extended by the authors ${ }^{4}$.

The crystallographic slip can take place whenever the resolved shear stress on a slip surface $\tau^{(\alpha)}$ assumes a value greater 
than the critical one, commonly named CRSS. The reference framework for the crystal plasticity constitutive theory is the one presented by Asaro and Lubarda ${ }^{5)}$ and by Pierce, Asaro and Needleman ${ }^{6), 7)}$. They expressed the CRSS by means of the unit vector defining the slip directions $\boldsymbol{s}^{*(\alpha)}$, the one normal to the sliding plane $\boldsymbol{m}^{*(\alpha)}$ (the subscript 0 indicates the reference configuration) and the Kirchhoff stress $J \sigma$ as shown in as in Eq.(2):

$$
\begin{aligned}
\boldsymbol{s}^{*(\alpha)} & =\boldsymbol{F}^{e} \boldsymbol{F}^{t} \boldsymbol{s}_{0}^{(\alpha)} ; \boldsymbol{m}^{*(\alpha)}=\boldsymbol{m}_{0}^{(\alpha)} \boldsymbol{F}^{t-1} \boldsymbol{F}^{e-1} ; \\
\tau^{(\alpha)} & =\left(\boldsymbol{s}^{*(\alpha)} \otimes \boldsymbol{m}^{*(\alpha)}\right): J \boldsymbol{\sigma}
\end{aligned}
$$

The total plastic work can be imagined as formed by the sum of the plastic works of each slip system, allowing to write:

$$
J \boldsymbol{\sigma}: \boldsymbol{l}^{p}=\sum_{\alpha=1}^{n} \tau^{(\alpha)} \dot{\gamma}^{(\alpha)} ; \quad \boldsymbol{l}^{p}=\sum_{\alpha=1}^{n}\left(\boldsymbol{s}^{*(\alpha)} \otimes \boldsymbol{m}^{*(\alpha)}\right) \dot{\gamma}^{(\alpha)}
$$

$\boldsymbol{l}^{p}$ is the plastic velocity deformation gradient (obtainable by taking the time derivative of Eq. (1)) and $\dot{\gamma}^{(\alpha)}$ is the crystallographic slip, assumed in accordance with $^{8}$ :

$$
\begin{aligned}
& \dot{\gamma}^{(\alpha)}=\dot{a}\left|\tau^{(\alpha)} / g^{(\alpha)}\right|^{n^{v}} \operatorname{sign}\left(\tau^{(\alpha)} / g^{(\alpha)}\right) ; \dot{g}^{(\alpha)}=\sum_{\beta=1}^{n} h_{\alpha \beta} \dot{\gamma}^{(\beta)} ; \\
& h_{\alpha \alpha}=h_{0} \operatorname{sech}^{2}\left|h_{0} \gamma /\left(\tau_{s}-\tau_{0}\right)\right| ; \quad h_{\alpha \beta}=q h_{\alpha \alpha}
\end{aligned}
$$

$\dot{a}$ is the reference slip system, $g^{(\alpha)}$ is the slip resistance of the $\alpha$ system, $n^{v}$ controls the strain rate dependency and $h_{\alpha \beta}$ is the hardening moduli.

As it was implicitly done for the crystal plasticity, it is also possible to assume that the phase transformation happens at a material point, where the total transformation deformation gradient, $\mathbf{F}^{t}$ in Eq.(1), can be thought as a linear combination of special deformation gradients, each of them associated with a geometrical pattern proper of the material variants, and coefficients $c$, which give the measure on how predominant is a variant respect to the others:

$$
\boldsymbol{F}^{t}=\sum_{p=0}^{m} \hat{\boldsymbol{F}}^{t(p)} c^{(p)}
$$

the index $p$, from 1 to $m$, represents the different variants, whereas the $p=0$ case defines the matrix.

The formulation of these $c^{(p)}(\boldsymbol{x}, t)$ multipliers passes through the Clausius-Duhem inequality for the definition of a functional form from which it is possible to derive the exact mathematical expression. These coefficients can be physically interpreted as multiplier of the matrix and variants fractions each of which expressing a different deformation pattern $\hat{\boldsymbol{F}}^{t(p)}$. In different words they represent a fraction of a unitary volume associated with a specific deformation mechanisms proper of the crystallographic structure of the material, otherwise called variants ${ }^{9), 10)}$. For a more complete description of the variables the reader is referred to ${ }^{11)}$.
It is worth mentioning that the numerical model is provided with a sort of activation energy; if the module of the thermodynamic driving force assumes a value bigger that this energetic threshold the transformation from the matrix to the variants, or from one variant to another, can take place. This aspect is quite relevant since it guarantees a stable state for the austenitic phase, which otherwise could transform under every conditions.

The expression of the Cauchy stress rate in the current configuration derived from the hypo-elasticity framework developed by the reference ${ }^{5)}$.

\section{Numerical models}

The purpose of this paper is to describe the behavior of the retained austenite transformation inside a martensitic body in order to better understand the effect of this mechanism in terms of stress/strain distribution and localization. In the following paragraphs two different numerical examples have been carried out to investigate the role of the shape of the inclusion and the crystal plasticity influence during the phase transformation. All the simulations have been realized by means of a user subroutine inside the commercial finite element code Abaqus v6.13-14 12 .

\subsection{Shape effect of the austenitic grain}

The first numerical example deals with the investigation of the shape effect of a retained austenite grain surrounded by an elastic martensitic body. Figure 2 displays the mesh used in the computation (C3D8R brick elements) and the boundary conditions adopted. The alignment of the transformable material, which dimensions are $\mathrm{L} / 3 \mathrm{xL} / 3$ in the squared case (i.e. CASE $A$ ) and $\mathrm{r}=\mathrm{L} / 6$ in the rounded one (i.e. $C A S E B$ ), is placed in the center of the sample and surrounded by a purely linear elastic body. The mechanical properties have been assumed accordingly to Yoshida et al.'s previous work ${ }^{4)}$.

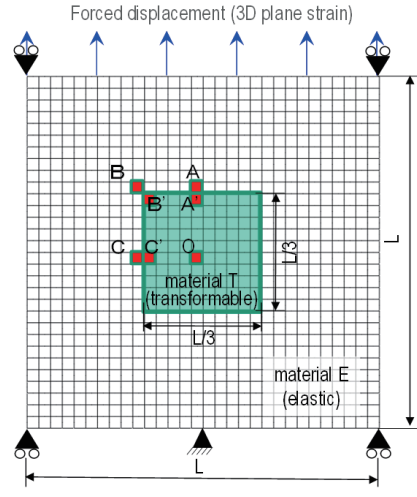

a)

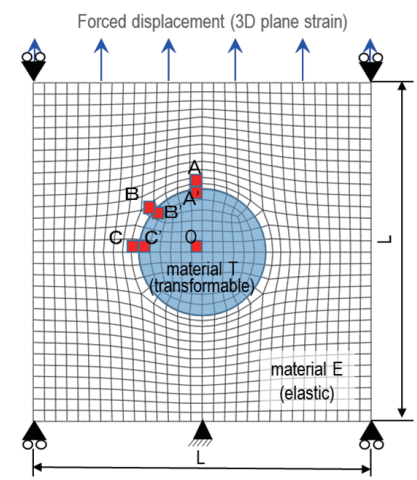

Fig. 2 (a) $C A S E A$ squared inclusion, (b) $C A S E B$ rounded inclusion. 
A uniaxial displacement controlled condition is applied on the top surface and the maximum elongation reaches the $12 \%$ of the nominal axial strain (i.e. $\Delta L / L$, where $L$ has been assumed as unitary since the purpose is merely a parametric study with no comparison with experiments). The displacements are constrained in depth, simulating a sort of 2D plane strain condition.

Equation (6) reports the total deformation gradient according to Morito et al. ${ }^{10)}$, which has been subjected to an averaging process in order to speed up the computation, reducing the number of variants from 24 to $6^{9)}$.

$$
F^{T}=\left(\begin{array}{rcc}
0.97578 & -0.03465 & -0.02422 \\
0.08515 & 1.12181 & 0.08515 \\
-0.08164 & -0.11677 & 0.91837
\end{array}\right)
$$

The crystallographic orientations and the elastic material properties remain unchanged for both materials. a)

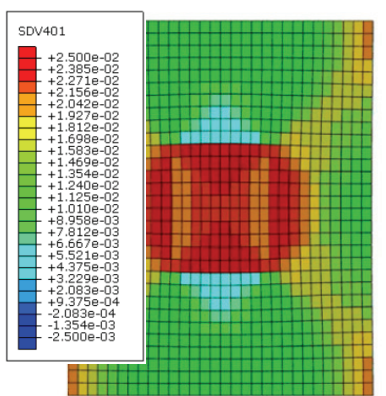

c)

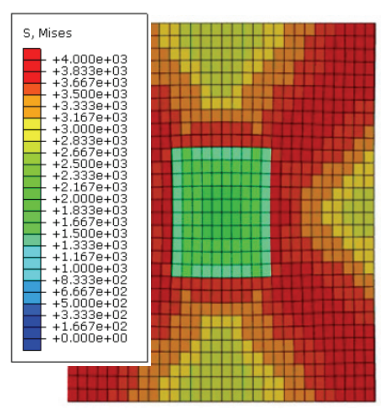

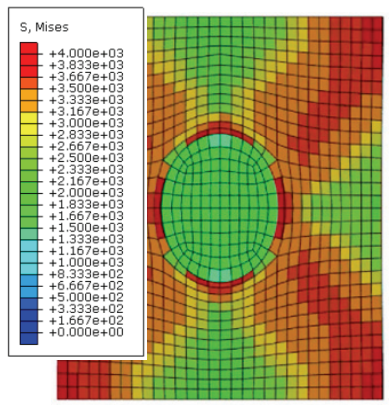

b)

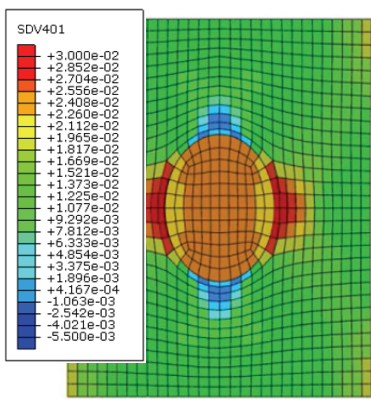

d)
Fig. 3 Mises stress (MPa) contour fields for the squared inclusion (a) and for the rounded one (b), volumetric strain contour fields for the squared inclusion (c) and for the rounded one (d).

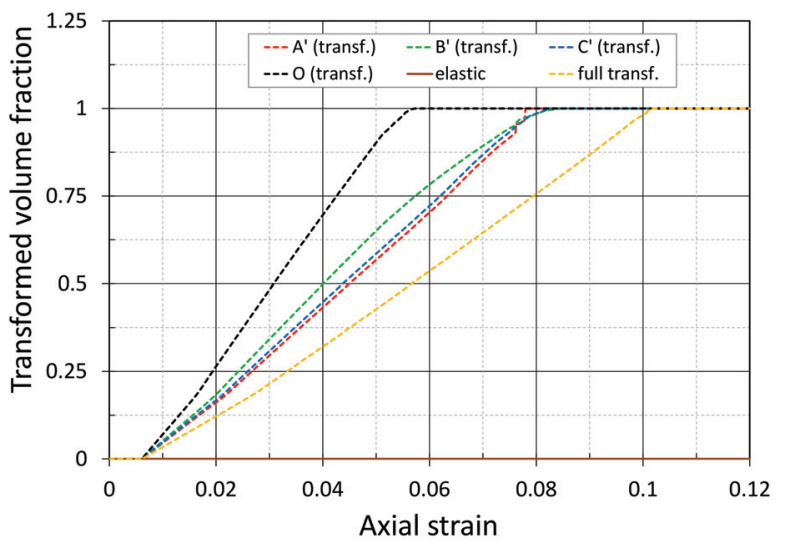

Fig. 4 Transformed volumetric fraction for the squared austenitic grain.
Observing Fig. 4 and Fig. 6 it is possible to point out few considerations: first of all that there is a substantial difference of the transformation speed for the element $\mathrm{O}$, placed in the middle of the inclusion, and the ones located at the border for the squared type inclusion. This can be attributed to an irregular distribution of the stress inside the transformable body, in fact all the sampling elements for the rounded grain display a much more uniform transformation speed. In addition the passage from austenite to martensite is completed around the $8 \%$ of the nominal axial deformation for the CASE $A$ against the $7 \%$ of CASE B.

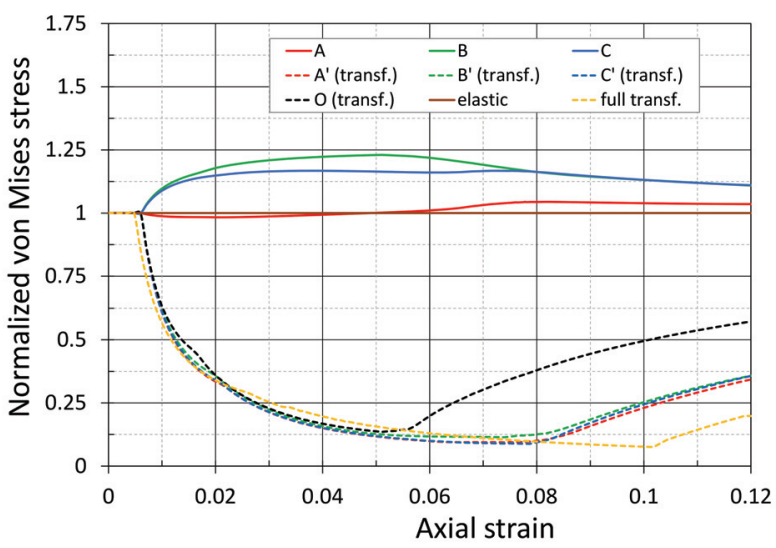

Fig. 5 Normalized Von Mises stress for the squared retained austenite.

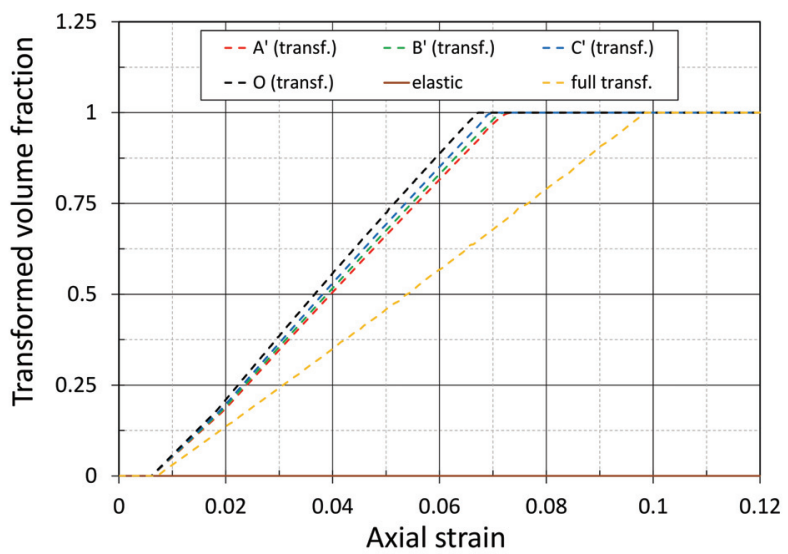

Fig. 6 Transformed volumetric fraction for the rounded austenitic grain.

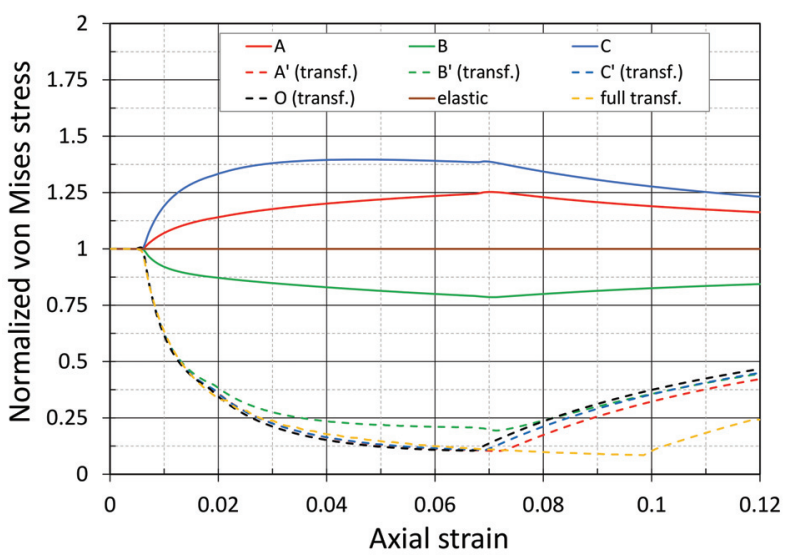

Fig. 7 Normalized Von Mises stress for rounded retained austenite. 
Focusing the attention on the Mises stress contour fields in Fig. 3 it is possible to appreciate how the maximum values of the stress are concentrated in few regions (upper, lower parts and on the two sides of the circular inclusion in the martensitic matrix) for the rounded grain, whereas they appear to be more uniformly distributed around the grain in the squared one. More in detail, looking at the Mises stress, normalized against the linear elastic solution in Fig. 5 and Fig. 7, it is possible to notice that for the squared grain case the maximum deviation from the reference is about $25 \%$, reached by the corner element B. The peak in Fig. 7 is much higher, around $37 \%$ for the lateral element $\mathrm{C}$, moreover element $\mathrm{A}$ has a $25 \%$ deviation exactly when the austenite completed the transformation into martensite. In addition it should be mentioned that, in this second scenario, the element $B$ shows a relaxation of the stress regime due to the redistribution of the stress. Figure $3 \mathrm{c}$ ) and d) report the volumetric strain for the two types of inclusion, drawing the attention on the effect of the corners, which cause an expansion of the elements along the diagonals in c), not visible in the rounded case.

\subsection{Effect of the crystal plasticity on the phase transformation}

The purpose of this second example is to compare the solution obtained activating or not the crystallographic slip, and therefore to investigate the effect of the crystal plasticity on the phase transformation in a polycrystalline sample.

Figure 8 displays the mesh with the 27 grains and the boundary conditions which are substantially the same of the previous example, with the only exception that in this case the displacements on top are carried until the $6 \%$ of the nominal axial deformation. Table 1 reports respectively the crystal plasticity parameters adopted for the computations according to Eq.(4), and the Euler angles for all the grains are taken accordingly to the reference $^{13)}$. The material parameters and the constraining condition along the thickness have been assume similarly to paragraph 3.1 analysis.
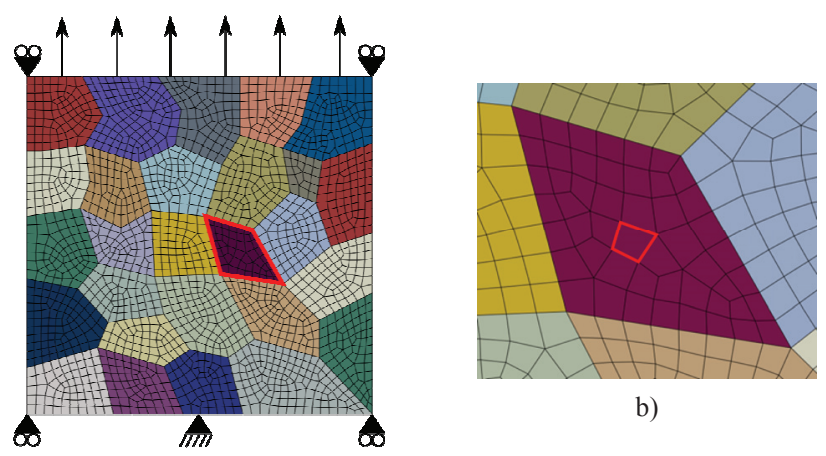

b)

a)

Fig. 8 (a) mesh used for the numerical simulation, (b) reference element for the variants volume fraction evolution.
Table 1 crystal plasticity parameters.

\begin{tabular}{ccccc}
$n^{v}$ & $\dot{a}$ & $h_{0}$ & $\tau_{s}$ & $\tau_{0}$ \\
\hline \hline 30 & 0.001 & 1020 & 300 & 93
\end{tabular}

For sake of simplicity it has been assumed that only the grain 11, highlighted in Fig. 8, is transformable, whereas the surrounding frame is purely elastic or elasto-plastic.

Observing the contour fields of Fig. 9 it is possible to notice how, considering an elasto-transformable body ET (i.e. Fig. 9a), the stress peaks tend to be distributed at the boundary of the grains and especially around the upper edge of the retained austenite. The same tendency is visible, in a lower amount, when crystal plasticity is taken into account; in this second case in fact the grain 11 is the only one showing a stress concentration. It has to be said that, besides some effects due to the phase transformation, the localization of the stress might be due to the geometrical shape of the inclusion together with its the set of Euler angles and the sets of the surrounding domains.

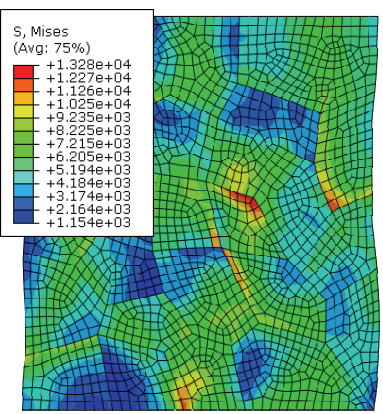

a)

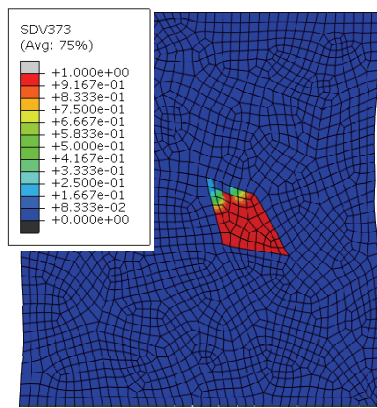

c)

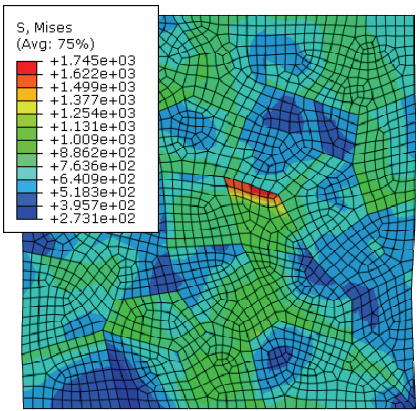

b)

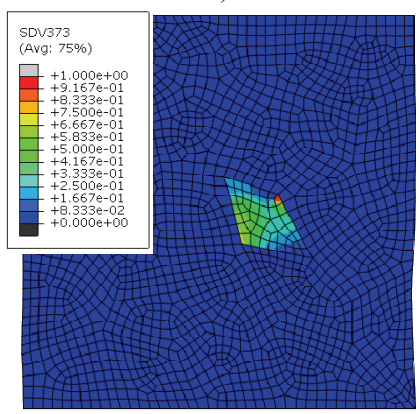

d)
Fig. 9 (a) Mises stress (MPa) contour field for the elasto-transformable (ET) body and for the elasto-plastic-transformable (ETP) one (b), (c) Transformation vole fraction for the ET and for the ETP (d).

Observing the curves shown in Fig. 10 it is quite clear that the stress regime is considerably reduced when the plastic slip is allowed as an additional deformation mechanism. The bifurcation between the light blue line and the red one (placed below the green) is localized slightly before the beginning of the grain transformation, affecting the transition phase. This aspect can be better appreciated having a look at the variants volume fraction evolution reported in Fig. 11 for the element in the middle of the 
retained body, as indicated in Fig. 8b.

Without the crystallographic slip (i.e. Fig. 11) the transformation happens very fast and it is completed just right after $1 \%$ of the nominal axial strain.

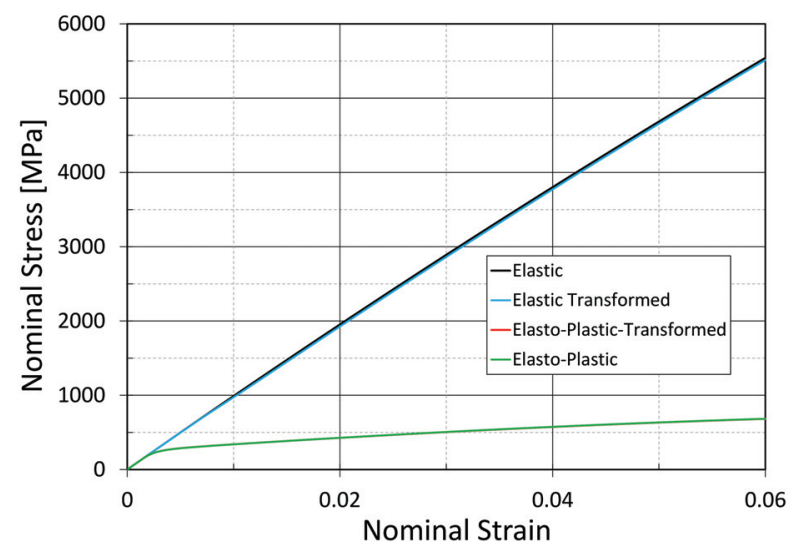

Fig. 10 Nominal stress vs nominal strain for the ET and EPT cases.

On the other hand, in the elasto-plastic-transformable case the total transformed volume fraction is just few percent at the same level of axial deformation and amounts to $40 \%$ at the maximum elongation. In the ET scenario all the variants are activated in equal measure, whereas in the EPT one just variant number 6 seems to be predominant over the others, proving once again the completely different stress configuration in the two cases.

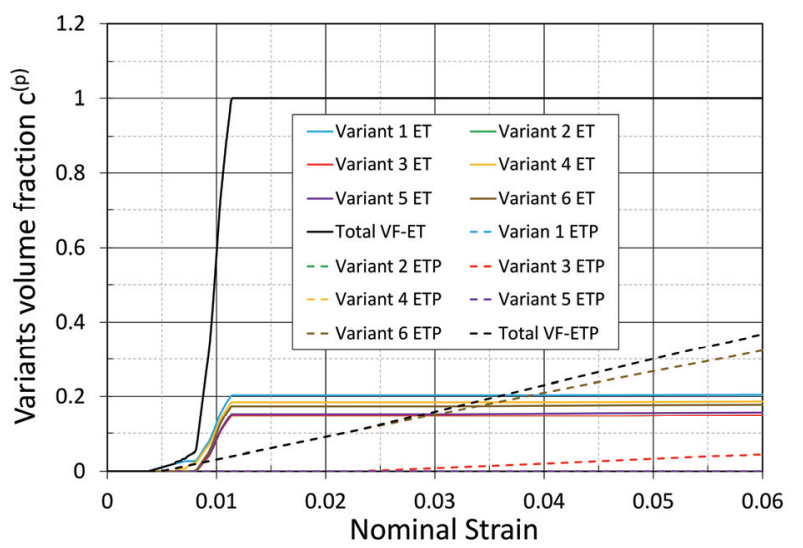

Fig. 11 Variants volume fraction evolution for the ET and ETP cases.

\section{Conclusions}

The MIMT phenomena is still nowadays object of several investigations due to the its complicate mechanism and to its role in modifying the material properties such toughness and ductility, not to mention that it also has been indicated as one of the possible responsible for the crack initiation.

The work presented in this paper aimed to study the local stress and strain distribution, the influence of the crystallographic slip and the different grain orientation effect by means of a thermodynamic single crystal constitutive model, capable of coupling together the crystal plasticity and the phase transformation. The finite element analyses carried out demonstrate that the geometry of the retained austenitic grain inside the elastic martensitic body affects both the stress regime and the volumetric strain in the surrounding of the inclusion. Moreover the phase transformation itself is influenced, displaying a faster and more uniformly distributed transition for a rounded shape. On the other hand the crystallographic slip, in the second numerical example, reduces sensibly the local stress peaks, inhibiting the transformation from the metastable austenitic phase to the martensitic one.

\section{Reference}

1) J.A. Kokosza, J. Pacyna, Effect of retained austenite on the fracture toughness of tempered tool steel, Arch. Mat. Scien. And Eng. ASME, 31(2) (2008), 87-90.

2) A. Mazur, J. Pacyna, T. Paluszkiewicz, J. Krawiarz, T. Skrzypek, The Influence of heat treatment parameters on susceptibility to cracking of hardener rolls. Report of AGH Metallurgy Institute 8/G (1978) (in Polish).

3) V.A. Idesman, I.V. Levitas, E. Stein, Elastoplastic materials with martensitic phase-transition and twinning at finite strains: numerical solution with the finite element method, Comput. Method. Appl. M. 173 (1999) 71-98

4) Y. Yoshida, et.al.; Strength prediction of cold-worked steel tubes by the crystal plasticity homogenizing method, Trans. JSCES, 14(2) (2009), 903-906.

5) J.R. Asaro, V.A. Lubarda, Mechanics of Solids and materials, Cambridge Univ. Press, NY, 2006.

6) D. Pierce, J.R. Asaro, A. Needleman, An Analysis of nonuniform and localized deformation in crystalline solids, Acta Metall. 30 (1982),1087-1119.

7) D. Pierce, J.R. Asaro, A. Needleman, Material rate dependence and localized deformation in crystalline solids, Acta Metall. 31 (1983),1951-1976.

8) J.R. Asaro, Crystal plasticity, J. Appl. Mech. 50 (1983), 921-934.

9) H.K. Yeddu, Martensitic Transformation in Steels - A 3D phase-field study, Phd Thesis, Stockholm, Sweden (2012).

10) S. Morito, H. Tanaka, R. Konishi, T. Furuhara, T, Maki, The morphology and crystallography of lath martensite in Fe-C alloys, Acta Materialia 51 (2003), 1789-1799.

11) V.A. Idesman, I.V. Levitas, D.L. Preston, J.Y Cho, Finite element simulations and microstructures based on a strain softening model, J. Mech. Pysics of solids, 53 (2005), 495-523.

12) Abaqus/Standard User Subroutines Reference Guide. DS. (2013).

13) Y. Shintaku, K. Terada, S. Tsutsumi, M. Kikuchi, Multi-scale crack propagation analysis considering polycrystalline plasticity deformation, CMD(2013), CD-rom. 